combination of physical characteristics makes the aldehyde a valuable and selective solvent, and both furfural and furfuryl alcohol find extensive use in the manufacture of synthetic resins. These applications are fully covered and a compilation of patents relating to furan resins is appended.

The book has many excellent features and is very well produced. It is authoritative, and, within the limits defined, comprehensive. It is to be regretted that the high price, possibly justified in the light of present-day production costs and the labour involved in its preparation, will severely restrict the sale of this most valuable treatise.

J. IDRIS JONES

\section{SCIENCE AND TECHNOLOGY OF TEXTILE FIBRES}

\section{Matthews' Textile Fibers}

Their Physical, Microscopic, and Chemical Properties. Prepared by a Staff of Specialists under the Editorship of Herbert R. Mauersberger. Sixth edition. Pp. $x+1283$. (New York: John Wiley and Sons, Inc. London: Chapman and Hall, Ltd., 1954.) £6 12s. net.

T $\mathrm{T}$ is just fifty years since the first edition of 1 Matthews's "Textile Fibers" appeared-a halfcentury that has witnessed the birth and rapid expansion of modern textile research, and the concurrent development of the technology of man-made fibres to its present size and importance. As was inevitable, during such a period, a steady stream of revisions has been necessary to keep such a fundamental reference work abreast of the times. The present edition, the sixth, worthily maintains the succession. As in the case of its immediate predecessor, it consists of a series of monographs, by distinguished American authors, which together cover the whole field of the textile fibres-natural, artificial and synthetic, their identification, properties, uses and assessment.

The weakest section is that devoted to fibre-testing methods, which remains practically unaltered from the previous edition. Whereas much of the early testing apparatus described has no modern interest and could have been omitted, no reference is made to the considerable volume of work that has been published on the bending properties of fibres, abrasion resistance, fatigue effects and creep phenomena. Of the ninety-five references contained in the bibliography of this section, only eight refer to work carried out within the past ten years. There is also a serious double transposition in the text on $\mathrm{pp}$. 1245-1247, which should be corrected in any future impressions.

In compensation, the section devoted to the microscopical and physical properties of wool, revised to include recent electron-microscopic studies, is excellent, as is also that dealing with the interpretation of X-ray fibre photographs, written for the non-specialist reader. This latter section appears for the first time in this edition. The sections concerned with man-made fibres have been considerably revised and extended. and, so far as the inevitable delays of publication allow, they are up to date. The need for a revision of these sections was, in fact, one of the chief reasons for the present edition, and it has been admirably met.

The book is well indexed, and the bibliographies appended to each section greatly enhance its value as a work of reference. As "a textbook for use in colleges and technical schools" in Great Britain, its strongly American flavour, inevitable in view of the general authorship, is a decided disadvantage. Nevertheless, it will continue to be used for reference purposes, in the future as in the past, because of the scope and competence of its contribution to textile knowledge.

\section{BIRATIONAL GEOMETRY}

\section{Methods of Algebraic Geometry}

By Prof. W. V. D. Hodge and Dr. D. Pedoe. Vol. 3, Book 5 : Birational Geometry. Pp. $x+336$. (Cambridge: At the University Press, 1954.) 40s. net.

THE scope of this book is more restricted than might have been expected from the title. It does not deal with birational geometry as a whole, for the fundamental properties discovered by the great Italian geometers have been entirely omitted. The reason for this is explained in the preface. The authors' intention is to explain methods, not results or a unified theory, and not all methods, but only those based upon the modern abstract algebra of rings, ideals and valuations. These methods are largely due to the recent work of O. Zariski. The reader is assumed to be already versed in the ordinary methods of algebraic geometry. On the other hand, no knowledge of abstract algebra is assumed, beyond that contained in the earlier volumes. It is a great convenience that the geometer will find in this volume and the earlier ones all the algebra he needs, though it entails that nearly half of this volume is algebra rather than geometry.

This volume contains four chapters. The first deals with the theory of ideals in a commutative ring, including prime and primary ideals, remainderclass rings, subrings, extension and quotient rings, modules, multiplicative theory of ideals, and integral dependence. The second chapter deals with the arithmetic theory of varieties and applies the results of the first chapter to geometry. Great stress is laid upon projectively normal varieties, which are used extensively in the more advanced parts of birational theory. The third chapter deals with the concept of abstract algebra known as the valuation of a function field. This generalizes the classical theory of a branch of an algebraic curve, and is of fundamental importance in the theory of birational transformations. The authors point out that many of the results could have been obtained more simply and elegantly by Weil's method, using what are called specializa. tions. However, it is unlikely that a complete theory of birational transformations can be built up without valuations, so geometers should study this method. Valuation theory can also be applied to the theory of numbers, but the account here is restricted to what is required for geometry.

The fourth and last chapter is entitled "Birational Transformations". Most of it is devoted to problems concerning multiple points. A problem still unsolved is whether we can obtain, for every algebraic variety, a birational transform without multiple points. Some light is thrown upon the structure of multiple points by Zariski's local uniformization theorem. No less than seventy pages are devoted to the proof and applications of this fundamental theorem. The volume concludes with two pages of bibliography and bibliographical notes, and an index.

H. T. H. Piaggio 